\title{
Erratum to: Impaired myocardial blood flow reserve in subjects with metabolic syndrome analyzed using positron emission tomography and $\mathrm{N}-13$ labeled ammonia
}

\author{
Hiroki Teragawa $\cdot$ Koichi Morita $\cdot$ Hiroki Shishido $\cdot$ \\ Nobuaki Otsuka $\cdot$ Yutaka Hirokawa \\ Kazuaki Chayama $\cdot$ Nagara Tamaki $\cdot$ Yasuki Kihara
}

Published online: 15 July 2010

(C) Springer-Verlag 2010

\section{Erratum to: Eur J Nucl Med Mol Imaging}

$$
\text { DOI 10.1007/s00259-009-1307-6 }
$$

Table 3 contained some incorrect data. The correct table is reproduced here in full.

The online version of the original article can be found at http://dx.doi. org/10.1007/s00259-009-1307-6.

H. Teragawa $(\bowtie) \cdot$ Y. Kihara

Department of Cardiovascular Medicine,

Hiroshima University Graduate School of Biomedical Sciences,

1-2-3 Kasumi,

Minami-ku, Hiroshima 734-8551, Japan

e-mail: hteraga@gmail.com

K. Morita $\cdot$ N. Tamaki

Department of Nuclear Medicine,

Hokkaido University Graduate School of Medicine,

Sapporo, Japan

H. Shishido $\cdot$ N. Otsuka $\cdot$ Y. Hirokawa

Hiroshima Heiwa Clinic,

Hiroshima, Japan

\section{K. Chayama}

Department of Molecular Science and Medicine,

Hiroshima University Graduate School of Biomedical Sciences,

Hiroshima, Japan 
Table 3 Relationship between MFR and other parameters

\begin{tabular}{|c|c|c|c|c|c|c|}
\hline \multicolumn{3}{|l|}{ Univariate analyses } & \multicolumn{4}{|l|}{ Multivariate analyses } \\
\hline \multirow[t]{2}{*}{ Factor } & \multicolumn{2}{|c|}{ MFR } & \multirow[t]{2}{*}{ Factor } & \multicolumn{3}{|c|}{ MFR } \\
\hline & $\mathrm{r}$ & $\mathrm{p}$ Value & & $\mathrm{F}$ value & $95 \% \mathrm{CI}$ & $\mathrm{p}$ Value \\
\hline & & & HOMA-IR & 4.7226 & -0.9212 to -0.0215 & 0.0408 \\
\hline Abdominal obesity & . & 0.1828 & Hypertension & 4.5855 & -0.8281 to -0.0133 & 0.0436 \\
\hline Elevated triglycerides & . & 0.3596 & Posterior wall thickness & 2.9044 & -0.5765 to 0.0564 & 0.1024 \\
\hline Low HDL-cholesterolemia & . & 0.8144 & Body mass index & 0.5697 & -0.1541 to 0.3303 & 0.4584 \\
\hline $\begin{array}{l}\text { Hypertension } \\
\text { Hyperglycemia }\end{array}$ & . & $\begin{array}{l}0.0255 \\
0.1377\end{array}$ & & & & \\
\hline Taking statin & . & 0.0921 & & & & \\
\hline Body mass index & -0.444 & 0.0204 & & & & \\
\hline Waist circumference & -0.353 & 0.0705 & & & & \\
\hline Fasting blood sugar & -0.359 & 0.0655 & & & & \\
\hline HOMA-IR & -0.507 & 0.0075 & & & & \\
\hline Hemoglobin A1C & -0.363 & 0.0624 & & & & \\
\hline Interventricular septal thickness & -0.367 & 0.0595 & & & & \\
\hline Posterior wall thickness & -0.417 & 0.0303 & & & & \\
\hline Visceral fat area & -0.361 & 0.0651 & & & & \\
\hline
\end{tabular}

HOMA-IR, homeostasis model assessment-insulin resistance; LDL, low-density lipoprotein; MFR, myocardial flow reserve. $\mathrm{r}^{2}=0.449$ 